Christopher B. Nelson

Hans-Ulrich Wittchen

Max Planck Institute of Psychiatry,

Clinical Psychology and Epidemiology

Unit, Munich, Germany

\title{
The EDSP: Setting the Stage!
}

Analyses of trends in the field of psychoactive substance use have shown a general increase in the frequency and severity of problems in most countries. The modern drug epidemics which started in the 1960s in industrialized countries have now spread worldwide with an enormous increase in the use of cocaine, designer drugs, other stimulants, cannabis, hallucinogens and opiates in addition to the use of multiple drugs (including alcohol). This epidemic has brought about an increase in the risk of mortality caused by substance-related risk-taking behavior, accidents, infections (including HIV and hepatitis), overdose and other physical diseases. The mental and physical disabilities and handicaps associated with substance use also seem to increase considerably.

Although these trends have generally been identified in many countries around the world, knowledge about the scope and size of the problem in specific countries and regions is sparse. With the exception of fairly comprehensive and diversified monitoring systems and long-term research initiatives in the US, most other countries and particularly Germany have been fairly slow in recognizing the importance of the drug problem and designing appropriate initiatives to learn more about culture, country and region-specific characteristics of drug use and abuse, associated risk factors and consequences.

Addressing these issues is a challenging and complex task, requiring concerted action on the regional, national and international level by politicians, the legal system and police, administrators, clinicians and researchers. This collaboration is essential because it is extremely unlikely that one country, region or institution alone is able to carry out the necessary basic, epidemiological and clinical

\section{KARGER}

Fax +4161306 1234

E-Mail karger@karger.ch www. karger.com

(C) 1998 S. Karger AG, Basel

1022-6877/98/0042-0005\$15.00/0

This article is also accessible online at: http://BioMedNet.com/karger
}

research needed to provide a sound scientific basis for a rationally planned action and intervention program against the drug epidemic. Further, understanding and intervening the rapidly changing international drug distribution 'highways' and patterns certainly call for more intense collaborations.

Examples for research areas where international collaboration is needed include (a) harmonization efforts with regard to the measurement of drug use, abuse and dependence as well as (b) monitoring strategies to allow comparison between various countries.

These two issues are essential to determine to what degree knowledge and insight collected in one country can be generalized or projected to other countries. International coordination in basic science is essential not only to improve our knowledge about the neurobiology and psychology of addictive behaviors but also about the natural history of drug use over life course, the delineation of stages of involvement in different drugs (including legal drugs such as nicotine and alcohol), degrees of involvement within drug classes and between drug classes (i.e. initiation, intermittent use, regular use, dependence, remission and relapse). Such natural history research require on the one hand longitudinal studies of individuals over time, on the other cohort sequential designs, in order to identify classes of probable causes, and the time they operate in an individuals life, as well as to differentiate among age, cohort and historical changes and differences. Despite the availability of numerous cross-sectional studies in various regions, it is still difficult to put the puzzle together, due to differences in the operationalization of key constructs, different designs and instruments, making 
comparisons a daunting task. As much such complex tasks should be part of an international network, we should acknowledge that cultural, country and regional characteristics play an extremely important role in many of these topics, ranging from the availability of psychotropic substances, over consumption patterns to possibly also the development of substance disorders. Social factors and norms, for example, have well been documented to be powerful predictors for the initiation and the experimental use of drug use, while intraindividual factors of neurobiological and psychological nature might be more important in influencing transitions to dependence and possibly abuse.

Among the high priority examples for action on the national and regional level probably the implementation of epidemiological studies along with continuous monitoring systems to determine ongoing changes in the availability of drugs, attitudes towards their use and actual use should be mentioned. In addition better epidemiological data are needed on the actual number of and the characteristics of addicts in a given area. Hence, the issue of recognition and diagnosis of substance use at all levels of health care has become of the utmost public health importance as a prerequisite for designing preventive and therapeutic interventions.

\section{Addressing These Issues: \\ The German Governments Research Initiative}

Many of these issues have been recognized for at least two decades, as discussed and summarized at the 1994 meeting of the College on Problems of Drug Dependence (CPDD, NIDA Res Monogr 151). But it took considerably longer in European countries to launch similarly comprehensive and coordinated action as in the USA, with the establishment of the European Monitoring Center for Drugs and Drug Addiction (EMCDDA) 1994 in Lisbon as the most impressive example.

In Germany, the Government presented, in 1990, a national drug initiative (Nationaler Rauschgift-Bekämpfungsplan) and as the consequence in 1993 a fairly comprehensive coordinated national multicenter research program, called 'Biological and Psychosocial Factors of Drug Abuse'. This research program encompasses a wide range of basic, applied and especially epidemiological projects that are the focus of this supplement.

The participants of the epidemiological projects are combined in a special work group named ANEPSA (Analytical Epidemiology of Substance Abuse) working with almost identical assessment instruments and coordinated design features. Four main projects constitute the core of this program. (1) In the northern German area of Lübeck a general population study of more than 4,000 community respondents investigates the lifetime and cross-sectional prevalence of substance use disorders in the population aged 18-64 with a linked longitudinal project on remission without formal interventions. (2) The Institut für Therapieforschung in Munich investigates the longitudinal course of risk cohorts of drug users to identify patterns of course as well as preventive strategies. (3) And finally the unit for Clinical Psychology and Epidemiology of the Max Planck Institute of Psychiatry in Munich studies prevalence and incidence of drug use among adolescents and young adults over a period of 5 years, in attempt to study early developmental stages of drug abuse with focus on the identification of developmental stages, risk factors and consequences. This study, named EDSP, also includes (4) a family genetic component as well as experimental neurobiological studies in subsamples.

\section{Early Developmental Stages of Substance Disorder Project (EDSP): Overview of the Baseline Findings}

In this issue, we take the opportunity to describe the background, the aims and the methods of the EDSP in more detail. Because the data analyses on the follow-up investigations as well as the supplementary studies are not yet completed, with the final follow-up wave being completed end of 1998, this overview will focus on the baseline findings.

Starting with a selected literature review of available epidemiological data on substance use and substance use disorders by Perkonigg and co-workers, we will present in detail our overall design and the specific design features [Wittchen et al.]. This paper will also provide a summary of statistical measures used. Since the assessment of substance use patterns and especially substance disorders is of key importance for our study, the paper by Lachner's group presents data on the reliability of our main study instrument, the M-CIDI, focussing on the substance section. The paper by Nelson's group opens the presentation of prevalence findings with a contribution on smoking and nicotine dependence. Although some readers might be surprised to see nicotine among the substance disorders, our findings will provide evidence, that smoking and nicotine dependence are not only of great general importance from a public health perspective, but are obviously 
also related systematically to other substance disorders as well as other forms of mental disorders. Wittchen and coworkers will present the findings on alcohol use and alcohol disorders, presenting unexpectedly high estimates of both abuse and dependence among our fairly young sample of 14- to 24-year-olds. The finding that in some cases according to our assessment strategy dependence syndromes of alcohol are existent even in fairly young age groups after very few years of exposure and regular use in fairly small amounts, will probably trigger some vivid discussions about the use of DSM-IV dependence criteria in young adolescents. Particularly the question how these findings match clinical diagnoses and how adequate diagnostic criteria for DSM-IV alcohol dependence are in ado- lescents remains an open issue for future research. Perkonigg's group summarizes the overall prevalence findings on illicit substance use and disorders. The fact that many of our respondents report frequently the use of various types of substances at the same time, resulting in a considerable number of cases in polysubstance disorders, led to the decision to report separately the findings of prescription drug use and abuse [Lieb et al.] and finally our results for ecstasy and related substances [Schuster et al.]. This final paper will probably receive considerable attention because of the ongoing debate, whether the prevalence of stimulants use is on the rise and whether these 'safe drugs' carry a substantial risk of developing abuse and dependence syndromes. 